\title{
Age-related Differences in Homocysteine and Serological Markers in Patients with Sudden Sensorineural Hearing Loss
}

\author{
Dehmet Burak Aşık1, (D) Erbil Kılıç², (D) Murat Bınar1 \\ 1 University of Health Sciences, Gülhane Training and Research Hospital, Clinic of Ear, Nose, Throat, Ankara, Turkey \\ 2istanbul Sultan Abdülhamid Han Training and Research Hospital, Clinic of Ear, Nose, Throat, istanbul, Turkey
}

\section{Abstract}

Objective: Sudden hearing loss is mostly a unilateral sensorineural hypoacusis with the highest incidence in young adults. The aim of this study was to determine the differences in homocysteine and serological marker levels in sudden sensorineural hearing loss and to define the importance of these markers in diagnosis.

Methods: After audiological examination, 52 patients were considered as having sudden hearing loss. Serological markers (C3, C4, anti-streptolysin-0, C-reactive protein, rheumatoid factor) and homocysteine were examined. The patients were divided into two groups, as patients above and below 40 years of age. Statistical analysis was performed using SPSS version 15 software. P values less than 0.05 were considered statistically significant.

Results: Fifty-two patients were included in the study, and the study group consisted of 33 men and 19 women. The mean age was 43.5 years and the age range was $14-82$ years. According to the survey results, $63 \%$ of the patients had higher than normal homocysteine levels. It was observed that $60.8 \%$ of homocysteine levels were increased in patients below 40 years of age and $69 \%$ in patients over 40 years of age. There was no statistically significant difference between the two groups ( $p=0.49$ ). In the evaluation of immunological serological markers, no parameter was observed except for C3c. Fifty-four percent of all patients were considered to have a C3c parameter below normal limits. C3c levels were decreased in 52\% of patients below 40 years of age and 55\% of patients over 40 years of age. There was no statistically significant difference between the two groups $(p=0.31)$.

Conclusion: It is thought that microvascular and immunological pathogenesis develops in sudden hearing loss. Homocysteine and C3 levels are valuable but agerelated parameters following diagnosis and treatment.

Keywords: Sudden sensorineural hearing loss, homocysteine, serology, prognosis

\section{INTRODUCTION}

Sudden hearing loss is a clinical condition that is frequently idiopathic and develops in the last 3 days. It is described audiologically as sensorineural hearing loss of more than 30 $\mathrm{dB}$ at least 3 consecutive frequencies (1). The incidence of the disease varies between 5-20 per 100,000 per year and peak incidence occurs between the ages of 41-50 years, with no difference between male and female genders (1-3). In addition, being over 40 years of age has been reported to have a negative effect (4). Vascular, autoimmune, infectious and intracochlear pathological processes have been implicated in the mechanism of the disease $(1,2,5,6)$. Although all of these processes play different roles in the pathophysiology, their effects on the cochlea cannot be determined biochemically and serologically
(5). Although vascular, autoimmune, infectious and intracochlear mechanisms have been held responsible, their role in the etiology has not been fully established. Changes in the levels of biochemical and serological markers provide information about the role of these theories in sudden hearing loss. In the literature, lipid profile and homocysteine were thought to play a role in microvascular processes (1). Similarly, in the literature, accompanying autoimmune diseases have been reported in many patients with sudden hearing loss $(7,8)$. The treatment and prognosis of sudden hearing loss depends on the accuracy of the diagnosis of the disease (9). Diagnosis is made by audiometry and clinical criteria in these patients, but it cannot be supported by biochemical and serological tests. The aim of this study was to determine the changes in the levels of immunological 
markers and acute phase reactants in sudden hearing loss and to determine the diagnostic value of these markers in the etiology of the disease.

\section{METHODS}

Serological markers and homocysteine results were evaluated retrospectively in patients who presented to our clinic with audiological and clinical diagnostic criteria of sudden hearing loss between 2011-2013 and had no organic cause of sudden hearing loss. Ethics committee approval was obtained from the Ethics Committee of Gülhane Training and Research Hospital (2012-1491-79-12/1648.3-4674). Written informed consent was obtained from the patients. Homocysteine and C3 parameters were analyzed by High-performance liquid chromatography (Shimadzu, HPLC, Kyoto, Japan). Laboratory reference values were 6-15 mmoL/L for homocysteine and 0.55-1.20 g/L for C3. Exclusion criteria were treatment history for current sudden hearing loss, sudden hearing loss due to other than idiopathic and acoustic trauma, steroid treatment due to comorbidities and other chronic diseases requiring treatment.

After audiological examinations, serological markers [C3, C4, ASO (anti-streptolysin-0), C-reactive protein (CRP), rheumatoid factor (RF)], and homocysteine were examined in patients who were considered as having sudden hearing loss. Steroid treatment was not given to the patients in order not to affect the levels of markers before taking blood for the tests. After obtaining blood, patients were treated according to their age, body weight, comorbidities and clinical conditions. The patients were divided into two groups and the groups were defined as patients above and below 40 years of age, which is considered to be important for prognosis.

\section{Statistical Analysis}

Statistical analyses were performed using SPSS version 15.0 (IBM, Chicago, Illinois, USA). The normality of the variables was analyzed by analytical methods. Descriptive analyses were expressed with mean for normally distributed variables. Resulting data were expressed as numbers and percentages. The proportional changes in homocysteine and C3 parameters were compared between groups using chi-square test. $P$ value less than 0.05 was evaluated as statistically significant.

\section{RESULTS}

Fifty-two patients were included in the study, and the study group consisted of 33 men and 19 women. The mean age was 43.5 years and the age range was $14-82$ years. The age-related data and the ratio of patients according to admission time are presented in Table 1. Table 2 presents the distribution of patients according to audiogram types. There was no history of trauma, co-morbidities, chronic treatment, family history of sudden hearing loss or previous sudden hearing loss. According to the results of the study, $63 \%$ of the patients had higher than normal homocysteine values. It was observed that homocysteine levels increased in $60.8 \%$ of patients below 40 years of age and in $69 \%$ of patients over 40 years of age. There was no statistically significant difference between the two groups $(p=0.752)$ (Table 3). In the evaluation of immunological serological markers, no change was observed in any parameter except C3c. In 54\% of all patients, C3c was found to be below normal limits. C3c levels were decreased in 52\% of patients below 40 years of age and $55 \%$ of patients over 40 years of age. There was no statistically significant difference between the two groups $(p=1.00)$ (Table 4). C3 values according to audiogram types are shown in Table 5.

Table 1. Data about the age and admission times of the patients

\begin{tabular}{|l|l|l|l|}
\hline \multicolumn{5}{|l|}{} & Male $(\mathbf{n = 3 3})$ & Female $(\mathbf{n = 1 9 )}$ & Total $(\mathbf{n = 5 2 )}$ \\
\hline Age & $15(45.4 \%)$ & $8(42.1 \%)$ & $23(44.2 \%)$ \\
\hline$<40$ years & $18(54.6 \%)$ & $11(57.9 \%)$ & $29(56.8 \%)$ \\
\hline$>40$ years & \multicolumn{5}{|l}{} \\
\hline Admission & $19(57.5 \%)$ & $13(68.4 \%)$ & $32(61.5 \%)$ \\
\hline$<7$ days & $14(42.5 \%)$ & $6(31.6 \%)$ & $20(38.5 \%)$ \\
\hline$>7$ days
\end{tabular}

Table 2. Data of patients according to audiogram types

\begin{tabular}{|l|l|l|l|}
\hline & $<40$ years & $>$ 40 years & Total \\
\hline Low & $5(21.7 \%)$ & $7(24.1 \%)$ & $12(23 \%)$ \\
\hline Flat & $2(8.6 \%)$ & $13(44.8 \%)$ & $15(28.9 \%)$ \\
\hline Total & $4(17.3 \%)$ & $2(6.8 \%)$ & $6(11.5 \%)$ \\
\hline High & $12(52.1 \%)$ & $7(24.1 \%)$ & $19(36.5 \%)$ \\
\hline
\end{tabular}

Table 3. Changes of homocysteine levels according to audiogram types

\begin{tabular}{|l|l|l|}
\hline Low & $11 / 12$ & $91.6 \%$ \\
\hline Flat & $11 / 15$ & $73.3 \%$ \\
\hline Total & $3 / 6$ & $50 \%$ \\
\hline High & $9 / 19$ & $47 \%$ \\
\hline
\end{tabular}

Table 4. Number of patients with change in C3 level

\begin{tabular}{|l|l|l|}
\hline$<\mathbf{4 0}$ years & $12 / 23$ & $52 \%$ \\
\hline$>\mathbf{4 0}$ years & $16 / 29$ & $55 \%$ \\
\hline Total & $\mathbf{2 8 / 5 2}$ & $54 \%$ \\
\hline
\end{tabular}




\begin{tabular}{|l|l|l|}
\hline \multicolumn{3}{|l|}{ Table 5. C3 levels according to audiogram types } \\
\hline Low & $6 / 12$ & $50 \%$ \\
\hline Flat & $8 / 15$ & $53 \%$ \\
\hline Total & $3 / 6$ & $50 \%$ \\
\hline High & $12 / 19$ & $63.1 \%$ \\
\hline
\end{tabular}

\section{DISCUSSION}

The aim of this study was to evaluate the microvascular and autoimmune processes responsible for the ethiopathogenesis of sudden hearing loss by laboratory tests and to evaluate the diagnostic utility of changes in markers. As there are changes in homocysteine and C3 levels in our study, we think that they can be used for diagnostic purposes before treatment in patients with sudden hearing loss.

In the literature, both biochemical and serological acute phase reactants have been questioned in studies investigating autoimmune mechanisms in sudden hearing loss and they have been reported to be useful in diagnosis (2). Greco et al. (7) and Werneck et al. (8) reported that C3 and C4 levels decreased but C3b levels did not decrease. Consistent with the literature, 54\% decrease was observed in C3 levels in our study. However, C4 levels did not change. According to Werneck et al. (8), these results support viral etiology. CRP and RF levels in our study contradict the literature. Toubi et al. (10) and Berrocal and Camacho-Ramirez (11) reported that CRP and RF levels increased and that this supported autoimmune etiology. In our study, no significant increase was detected in CRP and RF levels. Although there are sufficient publications in the literature supporting serological markers, these parameters may not be clinical and diagnostic indicators because sudden hearing loss is based on multifactorial mechanisms. Considering the reasons why CRP and RF values were not high in our patient group, it can be due to small number of patients in our study. Of course, although immunological theory is always valid for sudden hearing loss, these markers may not be elevated serologically. This may be due to the fact that patients were not carriers of any additional rheumatological and immunological diseases. Homocysteine is an amino acid that can be monitored in serum and that triggers pathological processes causing prothrombosis and hypercoagulability $(7,12)$. Diabetes, neural diseases and dyslipidemias frequently increase the level of homocysteine (13). The role of homocysteine in prothrombotic processes can be explained by cochlear vascular occlusion $(1,13)$. Plasma activator inhibitor-1 protein, anticardiolipin antibodies, lupus anticoagulants and lipoprotein (a) are also present in the formation of these pathological processes, and the same vascular pathological processes and markers are observed in cardiovascular diseases $(12,13)$. There are studies reporting 3-times higher risk of cardiovascular disease in patients with bilateral sudden hearing loss, and hypercoagulability and thromboembolism were held accountable $(13,14)$. Since $63 \%$ of the patients in our study had homocysteine levels above the normal range and homocysteine could be used as a cardiovascular risk test, we think that it can be used for routine controls. In our study, patients with cardiac disease were excluded, and we considered that homocysteine may be an appropriate marker for microvascular ethiopathogenesis in patients with sudden hearing loss due to the absence of statistically significant differences between patients above and below 40 years of age, but more studies should be performed on this issue. The limitations of our study were the low number of groups, the inability to form homogeneous groups when patients were classified according to audiogram types, and the presence of diseases that could change homocysteine and C3 levels but did not become clinically symptomatic. In addition, when we examined the statistical power of the study, the power of the study group consisting of 52 patients was $79 \%$ for C3 values and this power was calculated as $52.4 \%$ for homocysteine. It is important that the reader takes into account the power analysis results when evaluating the study results.

\section{CONCLUSION}

The diagnosis, treatment and follow-up of sudden hearing loss are based on the patient's history, physical examination and audiometric tests. Biochemical and serological markers cannot determine treatment response and prognosis, but are used only as supportive findings. As levels change independent of age after sudden hearing loss, we believe that homocysteine and C3 levels may be valuable parameters in diagnosis and treatment follow-up in sudden hearing loss with microvascular and immunological processes in the ethiopathogenesis. However, larger studies should be conducted on the changes of these parameters with age.

\section{Ethics}

Ethics Committee Approval: Ethics committee approval was obtained from the Ethics Committee of Gülhane Training and Research Hospital (2012-1491-79-12/1648.3-4674).

Informed Consent: Written informed consent was obtained from the patients.

Peer-review: Externally peer-reviewed.

\section{Authorship Contributions}

Surgical and Medical Practices: M.B.A., E.K., M.B., Concept: M.B.A., E.K., Design: M.B.A., E.K., Data Collection or Processing: 
M.B.A., E.K., Analysis or Interpretation: M.B.A., Literature Search: M.B.A., E.K., M.B., M.B.A., E.K., M.B., Writing: S.G., M.Y.

Conflict of Interest: No conflict of interest was declared by the authors.

Financial Disclosure: The authors declared that this study received no financial support.

\section{REFERENCES}

1. Cadoni G, Scorpecci A, Cianfrone F, Giannantonio S, Paludetti G, Lippa S. Serum fatty acids and cardiovascular risk factors in sudden sensorineural hearing loss: a case-control study. Ann Otol Rhinol Laryngol 2010;119:82-8.

2. Enache R, Sarafoleanu C. Prognostic factors in sudden hearing loss. J Med Life 2008;1:343-7.

3. Zhang X, Xu X, Ma W, Zhang Q, Tong B, Yu H, et al. A clinical study of sudden deafness. Acta Otolaryngol 2015;135:1030-5.

4. Wei X, He J. Analysis of prognostic factors for sudden sensorineural hearing loss. Lin Chung Erb Bi Yan Hou Tou Jing Wai Ke Za Zhi 2011;25:599-601.

5. Lasisi AO, Arinola AG. Acute Phase Reactants in Immune-Related Inner Ear Disease. Afr J Biomed Res 2010;13:113-7.
6. Suckfüll M. Perspectives on the Pathophysiology and Treatment of sudden Idiopathic Sensorineural Hearing Loss. Dtsch Arztebl Int 2009;106:669-76.

7. Greco A, Fusconi M, Gallo A, Marinelli C, Macri GF, De Vincentis M. Sudden Sensorineural Hearing Loss: An Autoimmune Disease ?. Autoimmun Rev 2011;756-61.

8. Werneck A, Gurgel L, Mello L, Albuquerque G. Sudden Sensorineural Hearing Loss: A Case Report Supporting the Immunologic Theory. Arq Neuropsiquiatr 2003;61:1018-22.

9. Wilson H, Alderson DJ. Sudden Sensorineural Hearing Loss: When is it Idiopatic?. J Laryngol Otol 2010;124:690-3.

10. Toubi E, Ben-David J, Kessel A, Halas K, Sabo E, Luntz M. Immune-mediated Disorders Associated with Idiopathic Sudden Sensorineural Hearing Loss. Ann Otol Rhinol Laryngol 2004;113:445-9.

11. Berrocal JR., Camacho-Ramirez R. Sudden Sensorineural Hearing Loss: Supporting the Immunologic Theory. Ann Otol Rhinol Laryngol 2002;111:989-97.

12. Ay M, Gürbilen M, Vatansev H. Akut Faz Proteinleri. Gen Tıp Der 1998;8:12432.

13. Marcucci R, Alessandrello Liotta A, Cellai AP, Rogolino A, Berloco P, Leprini E, et al. Cardiovascular and Thrombophilic Risk Factors for Idiopathic Sudden Sensorineural Hearing Loss. J Thromb Haemost 2005;3:929-34.

14. Oh JH, Park K, Lee SJ, Shin YR, Choung YH. Bilateral versus Unilateral Sudden Sensorineural Hearing Loss. Otolaryngol Head Neck Surg 2007;136:87-91. 